\title{
IDENTIFICATION OF NEW CATACLYSMIC VARIABLES IN THE 1RXS AND USNO-B1.0 CATALOGS
}

\author{
D. V. Denisenko ${ }^{1, *}$ and K. V. Sokolovsky ${ }^{2,3,{ }^{* *}}$ \\ ${ }^{1}$ Space Research Institute of Russian Academy of Sciences, Moscow, Russia \\ ${ }^{2}$ Max Planck Institute for Radio Astronomy, Bonn, Germany \\ ${ }^{3}$ Astro-Space Center, Lebedev Physical Institute of Russian Academy of Sciences, Moscow, Russia \\ As a result of applying the original optical variability search method on publicly \\ available data, we have found eight new cataclysmic variables and two possible Opti- \\ cally Violent Variable quasars among the previously unidentified X-ray sources in the \\ ROSAT catalog. We describe the search method and present the characteristics of \\ the newly identified variable stars. The obtained results demonstrate the large poten- \\ tial of the concept of Virtual Observatory for identifying new objects of astrophysical \\ interest.
}

\section{INTRODUCTION}

Despite the growing number of cataclysmic variable stars discovered both at optical wavelengths from the ground (see, for example, Wils et al., 2009, Drake et al., 2009) and in X-rays from space (Motch et al., 1996, Verbunt et al., 1997 and a number of other works), many of these objects, worthy of attention and further study, still remain undiscovered while the information about their unusual properties can be obtained from the existing data of various catalogs and archival observations. Following the concept of Virtual Observatory, we use simple search tools on the archival data which are publicly available for years to identify new objects of astrophysical interest.

Since 2005 one of the authors (D.D.) is carrying out a systematic work to identify the poorly studied X-ray sources from $1 \mathrm{RXS}$ catalog which is based on the data from ROSAT (Voges et al., 1999). As a result of this work several dozen new variable stars have been dis-

\footnotetext{
* Electronic address: denis@hea.iki.rssi.ru

** Electronic address: ksokolov@mpifr-bonn.mpg.de
} 
covered, including high-amplitude RS CVn stars (Denisenko, 2008), Algol type eclipsing binaries, as well as a few cataclysmic variables - deeply eclipsing polar 1RXS J020929.0+283243 (Denisenko et al., 2006), dwarf novae 1RXS J180834.7+101041 (Denisenko et al., 2008), 1RXS J173006.4+033813 (Denisenko et al., 2009), 1RXS J231935.0+364705 (Denisenko, 2009) and several others to be published. All these cataclysmic variables were identified in the archival images of DSS (Digitized Palomar Sky Survey photographic plates) with the occasional addition of data from the NEAT project devoted to asteroid search (Teegarden et al., 2003). DSS plates are covering the whole sky while NEAT data exist for the areas within about $40^{\circ}$ from the ecliptic, except for the Milky Way plane.

\section{VARIABILITY SEARCH METHOD}

Originally the search for variable objects was carried out in the vicinity of the 1RXS sources from the bright source catalog only (Voges et al., 1999) which lists 18806 objects. As of early 2010, we have checked about 700 northern hemisphere objects without identification in Simbad, NED and GCVS catalogs, according to the work by Zickgraf et al. (2003). Upon the discovery of the dwarf nova 1RXS J231935.0+364705 it was noted that the variability of this star could have been found on the basis of the USNO-B1.0 catalog data alone. Its outburst was detected in the infrared plate taken on 1995 August 28th, which is why it was included into the USNO-B1.0 catalog with magnitudes: $\mathrm{B} 1=17.73, \mathrm{R} 1=17.73, \mathrm{~B} 2=18.66$, $\mathrm{R} 2=17.73, \mathrm{I}=13.63$. Obviously the color indices $B-R=0.0, R-I=4.1$ do not correspond to any reasonable spectral energy distribution, even with the interstellar extinction taken into account and indicate the object's variability with an amplitude of about 4 magnitudes. The fortunate fact has been that the 2nd epoch (POSS-II) Palomar plates of the same areas of the sky but in different wavelengths, were taken on different dates. In fact the USNOB1.0 catalog contains the "light curves" in two passbands, although they consist of only two points, plus one point in the infrared band. Moreover, for about $60 \%$ of the northern celestial hemisphere in the areas where nearby plates are overlapping, there are several (2 to 4 ) images in each of the three bands $B, R$ and $I$. Thus we can detect the object's variability both by comparing their magnitudes at different epochs (B1 vs. B2, R1 vs. R2 in the USNO-B1.0 catalog) and by analyzing their $B-R$ and $R-I$ color indices, as well as by visual inspection of the photographic plates taken on different dates in the same wavelengths. 
Given that the 1st epoch (POSS-I) Blue and Red Palomar plates were taken during the same night immediately one after the other, the values of blue and red magnitudes B1 and R1 can serve as a good estimate of the color index $(B 1-R 1)$ even for the variable stars and non-stellar objects in the USNO-B1.0 catalog. This is an important criterion which allows us to separate the cold red $(B-R>3)$ variable stars of semiregular and Mira types (which can also have variability with an amplitude up to 2 or more magnitudes), from the systems of our interest with a blue dwarf or quasi-stellar objects (quasars) who have blue or white color indices $(B-R \sim 0)$. For example, in the case of 1RXS J231935.0+364705 the value of $\mathrm{B} 1-\mathrm{R} 1=0.0$ excludes the Mira scenario and along with a proper motion of about 14 mas per year it allows us to unambiguously identify it as a Galactic cataclysmic variable star.

In order to search for the variable objects of our interest in the USNO-B1.0 catalog (Monet et al., 2003) we have chosen the following criteria:

- declination $\delta>-10^{\circ}$;

- distance from an X-ray source listed in the ROSAT Bright (Voges et al., 1999) or Faint (Voges et al., 2000) catalogs $d<30^{\prime \prime}$;

- $12<\mathrm{R} 2<20$;

- $\mathrm{B} 1-\mathrm{R} 1<1.0$;

- $|\mathrm{B} 1-\mathrm{B} 2|>2.0$ or $|\mathrm{R} 1-\mathrm{R} 2|>2.0$ or $|\mathrm{R} 2-\mathrm{I}|>2.0$;

- "star/galaxy" discriminator in USNO-B1.0 is $\geq 7$;

- there are no bright stars in the immediate vicinity of the object;

- object is not present in the 2MASX catalog of extended infrared sources (mostly galaxies, Skrutskie et al., 2006);

- object is not present in the NVSS catalog of radio sources (Condon et al., 1998);

- object is not a known variable star, i.e. it is not listed in the General Catalogue of Variable Stars (Samus et al., 2007-2009) or the Variable Star Index (VSX, Watson et al., 2006).

The galactic cataclysmic variables and quasars have virtually the same $B-R$ and $R-I$ color indices, as well as very similar X-ray to optical luminosity ratios. Additional data can help with distinguishing them from each other: (a) proper motions reported in the USNOB1.0 catalog. Obviously quasars should have zero proper motion. If the object has a reported proper motion of $\sim 10$ mas/year or larger, one can tell with a high level of confidence that 
this is a star of our Galaxy. However, quite faint and distant stars can have proper motions too small to be detected even in $\sim 40$ years having passed between the 1st and 2nd epoch Palomar plates. (b) 2MASS infrared sky survey data (Skrutskie et al. 2006): the infrared $J-K_{s}$ color index of quasars is usually exceeding 1.0 unlike the stars whose $J-K_{s}$ lies in the range from 0.0 to 1.0 (see Figure 1 in Gänsicke et al., 2005). In a number of cases this allows to make the final choice in favor of one or the other nature of the object in question. However, additional photometric and/or spectroscopic observations may be required for the faint sources (generally with $R>18$ ) that are absent in 2MASS catalog. We have found two such objects in our work that do not have a proper motion in USNO-B1.0 and are absent in 2MASS. We have designated them as objects of unknown nature (QSO?).

The X-ray spectral information available in the 1RXS catalog in the form of hardness rations HR1 and HR2 (Voges et al., 1999; He et al., 2001) may provide additional clues about the nature of the sources. For a discussion of typical X-ray spectral parameters of cataclysmic variables see Verbunt et al. (1997).

Table 1. New variable stars identified with X-ray sources from 1RXS catalog.

\begin{tabular}{ccrcll}
\hline \hline 1RXS & USNO-B1.0 & $\mathrm{d}^{\prime \prime}$ & Range & Constellation & Type \\
\hline J043445.0+030623 & $0931-0068625$ & 11 & $17.9-20.0 R$ & Taurus & QSO? \\
J072103.3-055854 & $0840-0137592$ & 27 & $15.7-18.9 B$ & Monoceros & CV \\
J164103.6+784307 & $1687-0061156$ & 19 & $17.8-20.0 R$ & Ursa Minor & CV? \\
J174320.1-042953 & $0855-0326594$ & 4 & $15.1-18.1 R$ & Ophiuchus & CV \\
J184542.4+483134 & $1385-0291789$ & 3 & $16.6-21.3 B$ & Draco & QSO? \\
J184543.6+622334 & $1523-0313957$ & 13 & $16.9-19.7 B$ & Draco & CV \\
J185310.0+594509 & $1497-0258111$ & 4 & $18.5 R-15.4 I$ & Draco & CV \\
J192926.6+202038 & $1103-0421031$ & 17 & $16.6-19.0 R$ & Vulpecula & CV \\
J194151.4+752621 & $1654-0092633$ & 9 & $17.8-20.5 B$ & Draco & CV \\
J222335.6+074515 & $0977-0743560$ & 22 & $18.0-20.5 B$ & Pegasus & CV \\
\hline
\end{tabular}


Table 2. Magnitudes of the newly identified variable objects in the USNO-B1.0 catalog.

\begin{tabular}{cccccc}
\hline \hline USNO-B1.0 & B1 & R1 & B2 & R2 & I \\
\hline $0931-0068625$ & 18.57 & 17.86 & 19.49 & 19.96 & 18.20 \\
$0840-0137592$ & 15.71 & 15.17 & 18.88 & 16.78 & 16.80 \\
$1687-0061156$ & 18.47 & 17.82 & 20.87 & 19.99 & - \\
$0855-0326594$ & 16.12 & 15.15 & 18.95 & 18.13 & 17.10 \\
$1385-0291789$ & 21.32 & 19.81 & 16.61 & 19.47 & 19.04 \\
$1523-0313957$ & 16.88 & 17.28 & 19.66 & 19.11 & 18.52 \\
$1497-0258111$ & 17.75 & 18.66 & 17.87 & 18.51 & 15.44 \\
$1103-0421031$ & 17.53 & 16.60 & 20.13 & 19.02 & 16.62 \\
$1654-0092633$ & 17.84 & 18.25 & 20.46 & 19.55 & 18.22 \\
$0977-0743560$ & 17.95 & 17.72 & 20.50 & 17.60 & 17.63 \\
\hline
\end{tabular}

\section{SEARCH RESULTS}

Having applied the above criteria to the faint objects of 1RXS catalog we have obtained a list of 1460 candidates from USNO-B1.0. The analysis of their distribution over the celestial sphere has shown that over $85 \%$ of candidates are concentrated in several localized areas and are artifacts of the erroneous zero-point calibration for one of the catalog's stellar magnitudes. Besides their proximity in the sky, such objects have the same color index (for example, B1 - R1 or B2 - R2) outside the usual range. After removing such cases, a list of 203 candidates remains. Each of them was visually inspected using the digitized Palomar plates: 6 objects turned out to be "ghosts" from the nearby bright stars of 4-8m within 1-6', 4 were found to be plate defects, 3 were caused by the absence of 1 st epoch Blue plate, 31 are galaxies, 10 are quasars $\left(J-K_{s}\right.$ from 1.3 to 2.0$), 99$ are faint stars likely with the photometry error in one of filters due to the erroneous zero-point during the digitizing, 31 are pairs and groups of 3-5 nearby stars, 2 are "star + galaxy" pairs, 5 are high proper motion stars, 1 is a "white dwarf + red dwarf" pair (see below), 1 is a known cataclysmic variable discovered by Catalina Sky Survey on 2009 September 18th and 10 are previously unknown variable objects, 8 of those are cataclysmic variables and 2 are likely optically violent variable quasars. 
Dwarf novae stay at minimum light (quiescence) for 80 to $99 \%$ of the time depending on the variability subtype, hence the probability of detecting their variability using five stellar magnitudes of the USNO-B1.0 catalog varies significantly. One can estimate that about 25 to $40 \%$ of UGSU-type dwarf novae, which are in outburst phase from 5 to 10 per cent of the time, could be found using this method.

Among bright ROSAT sources (with ROSAT/PSPC count rate above 0.05 cts/s) we have found two new variable objects - 1RXS J184542.4+483134 in Draco and 1RXS J174320.1-042953 in Ophiuchus. The former is probably a quasar which underwent a flare in 1990 during the ROSAT observations and gradually faded away over the next years, and the latter - a cataclysmic variable. Details for these objects are given in the following section. The whole list of the newly discovered variables is presented in Table 1.

\section{INDIVIDUAL OBJECTS}

1RXS J043445.0+030623 = USNO-B1.0 0931-0068625: R.A.= 04:34:44.443, Dec. $=+$ 03:06:16.18, J2000, proper motion (hereafter $\Delta$ R.A., $\Delta$ Dec.): $(0,0)$ mas/year. Bai et al. (2007) mention this object among the Active Galactic Nuclei candidates but with no spectroscopic or photometric detail. The object is $2.1 \mathrm{~m}$ brighter on the 1955 Red Palomar 1st epoch plate than on the corresponding 1990 image (see Fig. 1). Zero proper motion in the USNO-B1.0 catalog as well as lack of soft emission in X-ray band (hardness according to 1RXS catalog is HR1 $=1.00 \pm 0.43, \mathrm{HR} 2=0.83 \pm 0.53)$ favor quasar nature of the object.

1RXS J072103.3-055854 = USNO-B1.0 0840-0137592: R.A.= 07:21:02.865, Dec. $=-05: 59: 20.42$, J2000, proper motion: (0, 0) mas/year. Despite the zero proper motion in the USNO-B1.0 catalog, the galactic latitude $b=+3.8$ and the absence of galaxies and their clusters in the vicinity (Fig. 2) favor the galactic nature of the object.

We have checked 22 photographic plates of the Moscow archive at the Sternberg Astronomical Institute of Moscow University (area SAO 134264) taken between November 1986 and February 1994. The star is not visible on 18 of them (fainter than the plate limit of 17.0-17.5 B), however it has photographic (blue) magnitude of $16.2-16.3$ on two plates of 1986 November 30th, 16.5 on the 1992 April 3rd plate and about 17.0 on 1993 January 19th. The star is likely a dwarf nova.

1RXS J164103.6+784307 = USNO-B1.0 1687-0061156: R.A.= 16:40:57.850, 


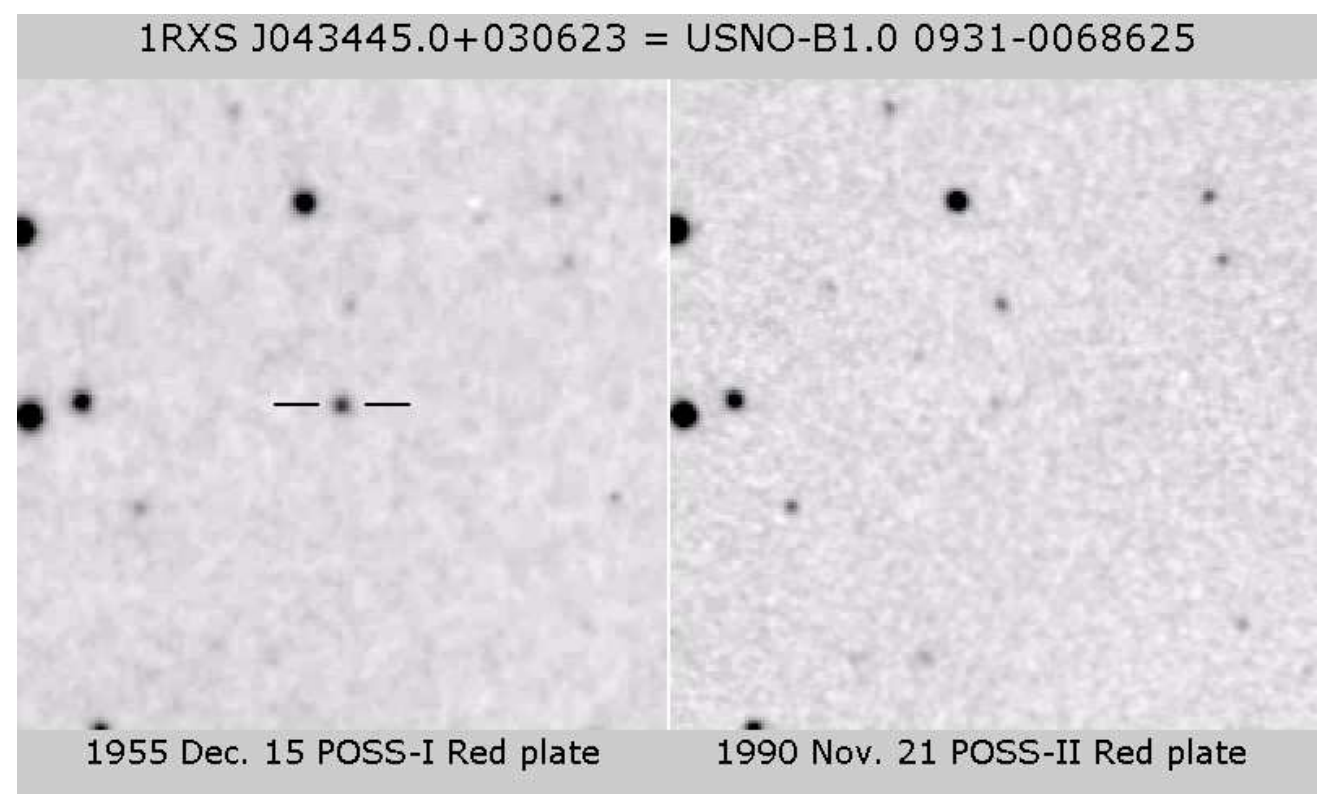

Figure 1. Finder chart of the source 1RXS J043445.0+030623 $\left(200^{\prime \prime} \times 200^{\prime \prime}\right)$.

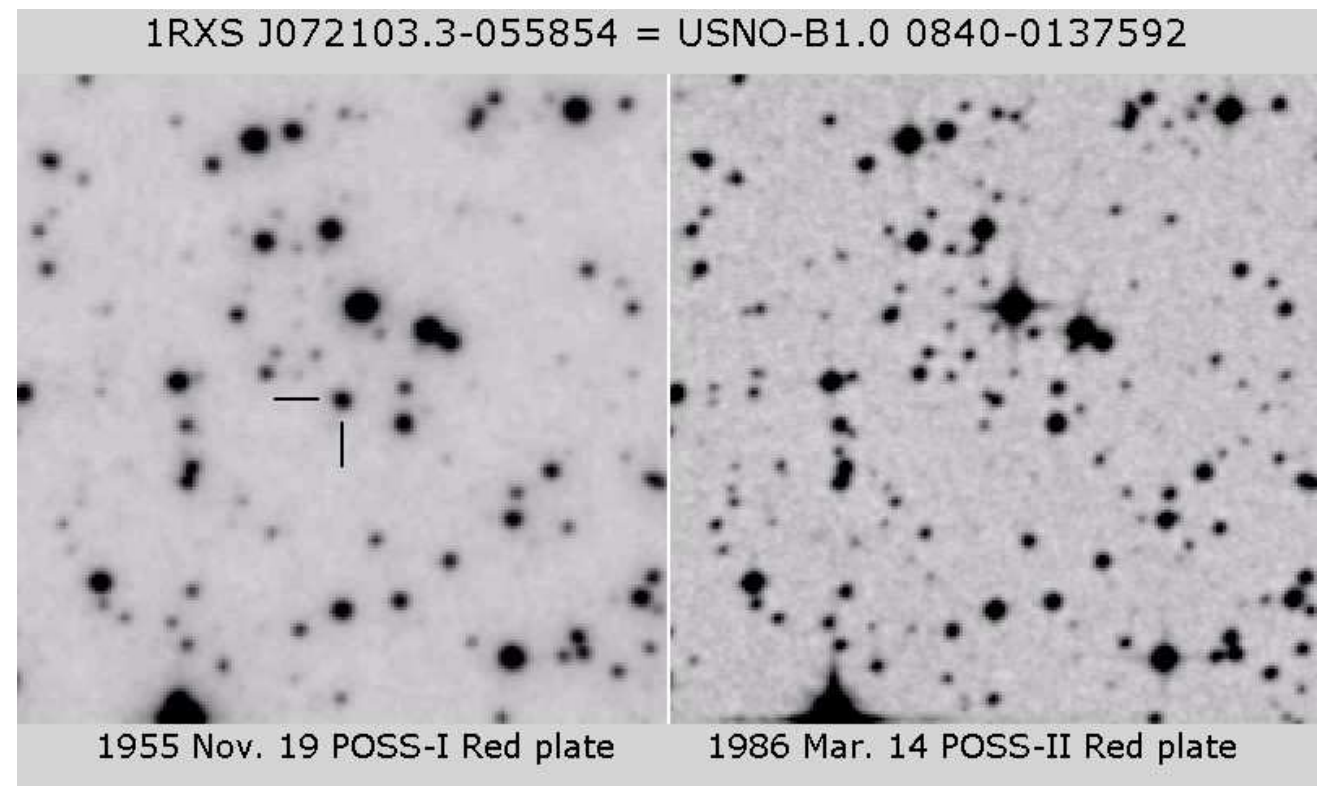

Figure 2. Finder chart of the source 1RXS J072103.3-055854 $\left(200^{\prime \prime} \times 200^{\prime \prime}\right)$. Note the faint star to the North-East of the variable at minimum light on the 1986 image.

Dec. $=+78: 42: 59.26$, J2000, proper motion: $(0,0)$ mas/year. There are faint galaxies present in the vicinity of the object (Fig. 3). It is possible that the object is actually a quasar. The hardness in X-ray band according to the ROSAT data (HR1 $=0.74 \pm 0.25, \mathrm{HR} 2=0.26 \pm 0.33$ ) does not allow to make an unambiguous conclusion about the object's nature. The follow-up 
observations are necessary.

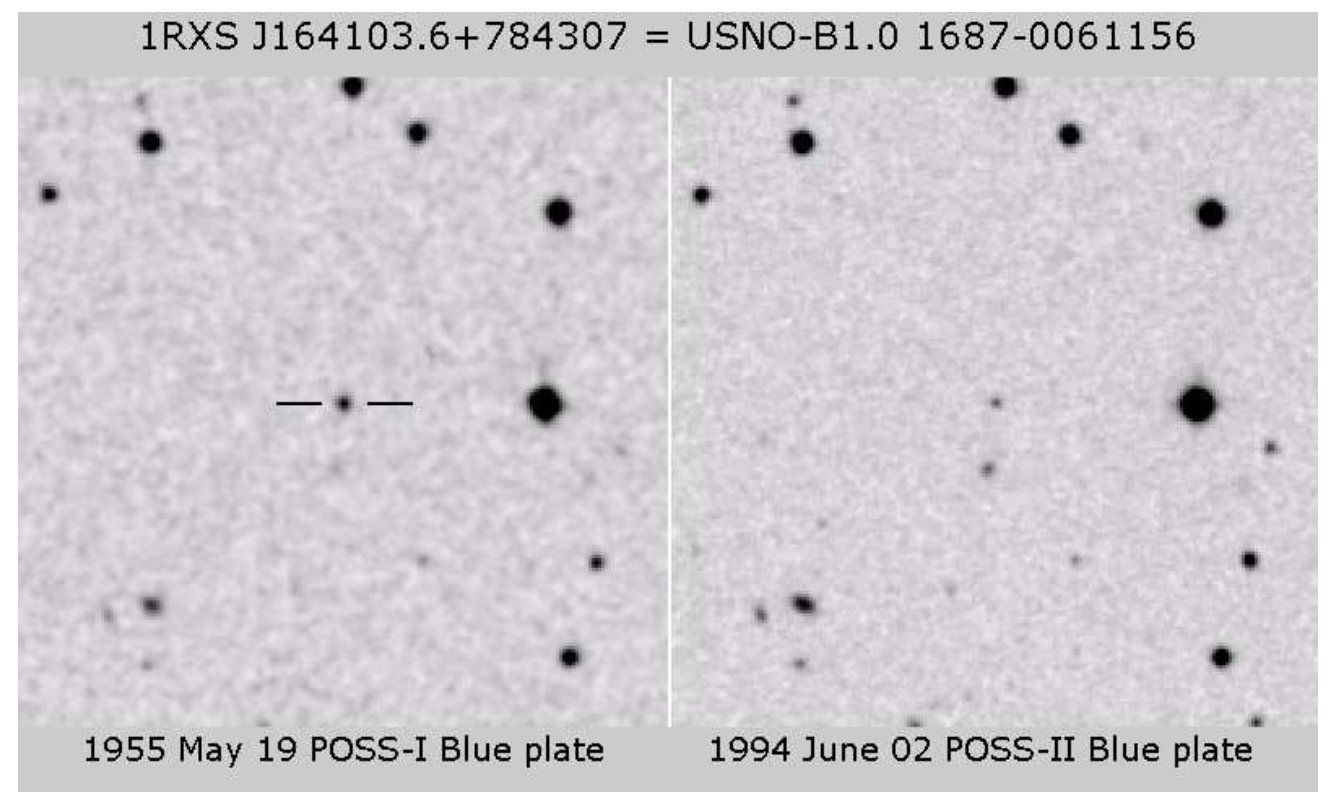

Figure 3. Finder chart of the source 1RXS J164103.6+784307 $\left(200^{\prime \prime} \times 200^{\prime \prime}\right)$.

1RXS J174320.1-042953 = USNO-B1.0 0855-0326594: R.A.= 17:43:20.287, Dec. $=-04: 29: 57.09$, J2000, proper motion: (18, -16) mas/year. Star is in outburst on the Blue and Red POSS-I Palomar plates (1954 July 1st, Fig. 4). Color index $J-K_{s}=0.64$ (according to the 2MASS catalog) and the proper motion clearly indicate that the object is a cataclysmic variable.

1RXS J184542.4+483134 = USNO-B1.0 1385-0291789: R.A.= 18:45:42.622, Dec. $=+48: 31: 30.84$, J2000, proper motion: $(0,0)$ mas/year. This source is of special interest. The object was at outburst on the Blue POSS-II Palomar plates taken on June 26th and July 21st, 1990 then gradually faded during several years. The source is still clearly seen on the Red plate of 1991 August 7th, while on 1992 August 26th it is already at the limit of sensitivity. The duration of outburst maximum (no less than 25 days) and monotonous fading of magnitude during more than two years are hardly consistent with the behavior of cataclysmic variables. Most likely the object is a quasar with large amplitude of variability (at least $5 \mathrm{~m}$ ), and ROSAT observations were coincident with its flare. The extragalactic nature of this source is also supported by the parameters of X-ray hardness in 1RXS catalog $(\mathrm{HR} 1=0.03 \pm 0.15, \mathrm{HR} 2=0.26 \pm 0.19)$ which are quite typical for quasars. The finding chart of the object is presented on Fig. 5. 


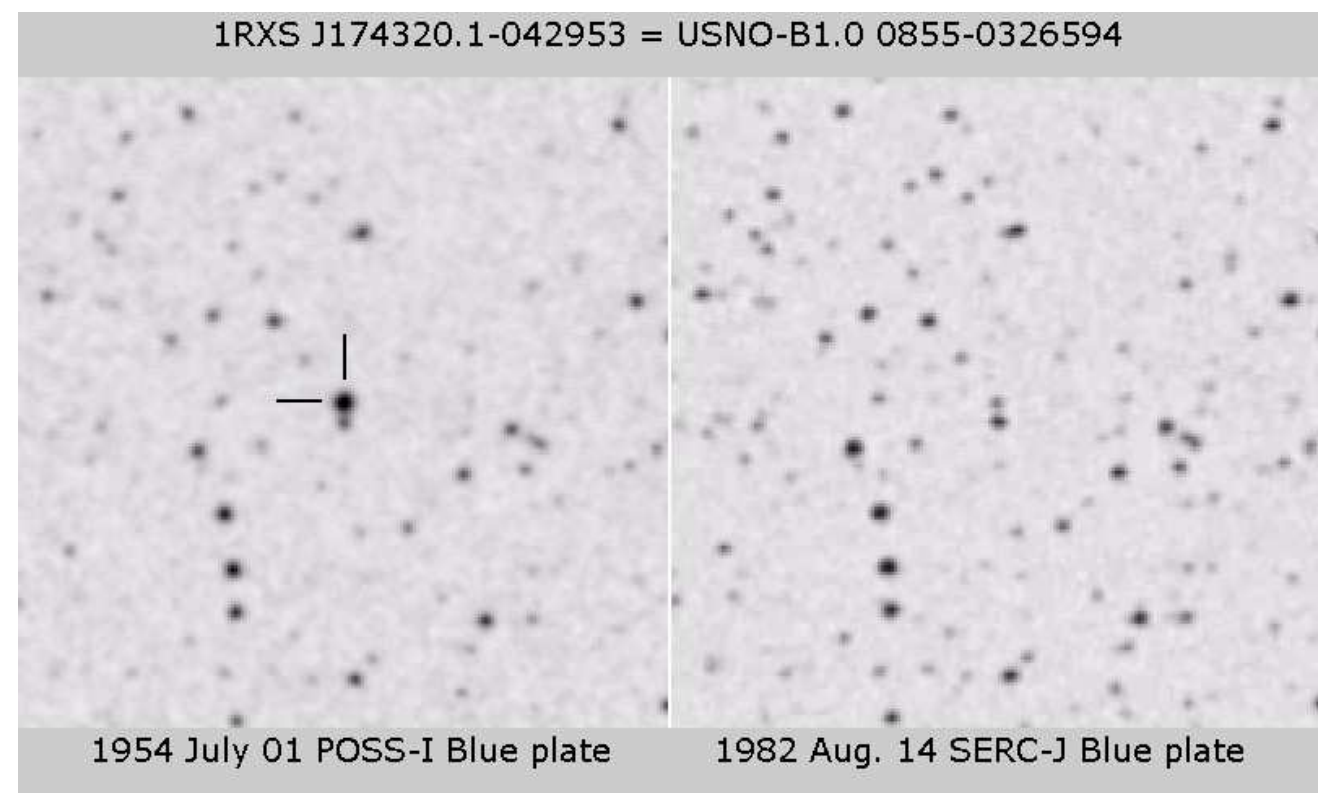

Figure 4. Finder chart of the source 1RXS J174320.1-042953 $\left(200^{\prime \prime} \times 200^{\prime \prime}\right)$. Note the faint star $6^{\prime \prime}$ due South of the variable.

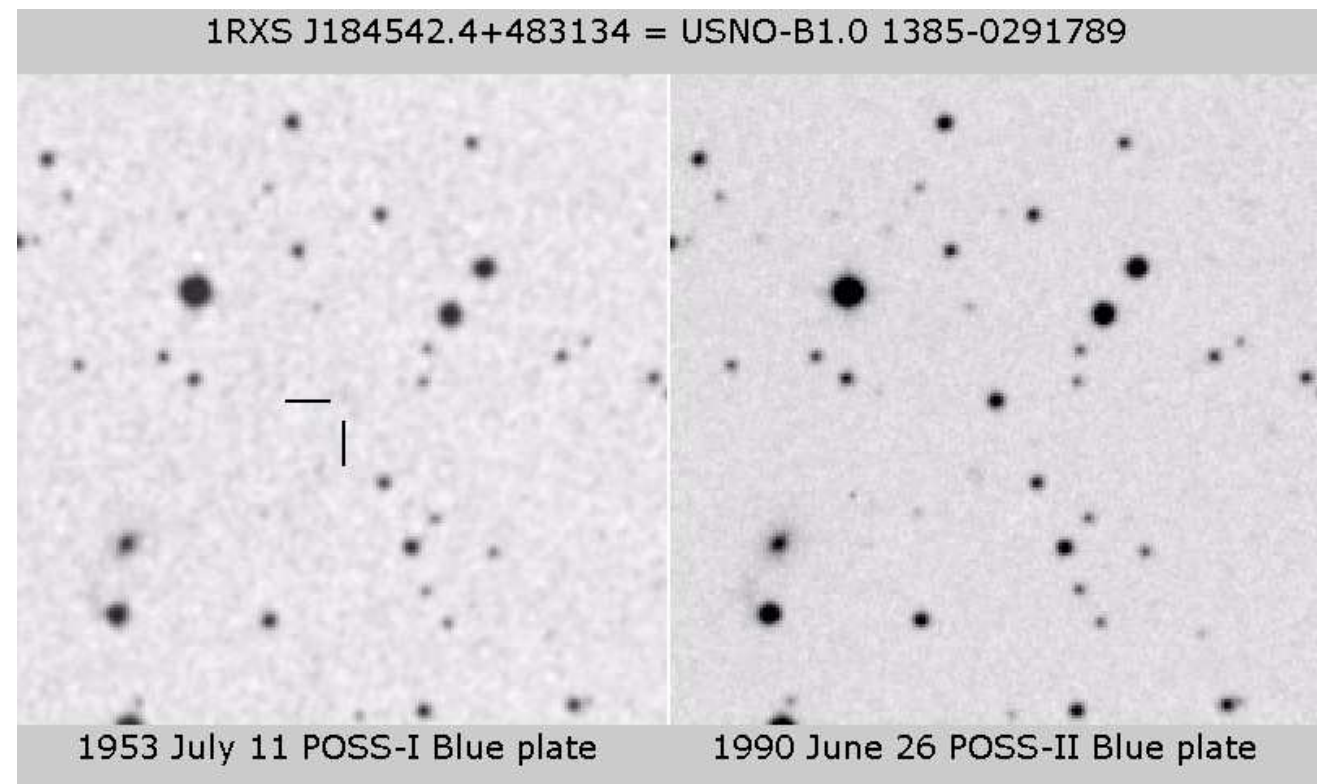

Figure 5. Finder chart of the source 1RXS J184542.4+483134 $\left(200^{\prime \prime} \times 200^{\prime \prime}\right)$. On the 1953 image (left panel) the object is not visible, its position being marked with dashes. Note the galaxy 1.4 South-East (to the bottom left) of the source.

1RXS J184543.6+622334 = USNO-B1.0 1523-0313957: R.A.= 18:45:42.285, Dec. $=+62: 23: 43.99$, J2000, proper motion: $(-2,8)$ mas/year. The hardness parameters in $1 \mathrm{RXS}$ catalog $(\mathrm{HR} 1=0.52 \pm 0.23, \mathrm{HR} 2=0.40 \pm 0.21)$ are corresponding to the values 
characteristic of the magnetic cataclysmic variables (for the polar 1RXS J005528.0+461143 they are equal to $0.37 \pm 0.19$ and $0.53 \pm 0.20$, respectively). The finding chart of the object is presented on Fig. 6.

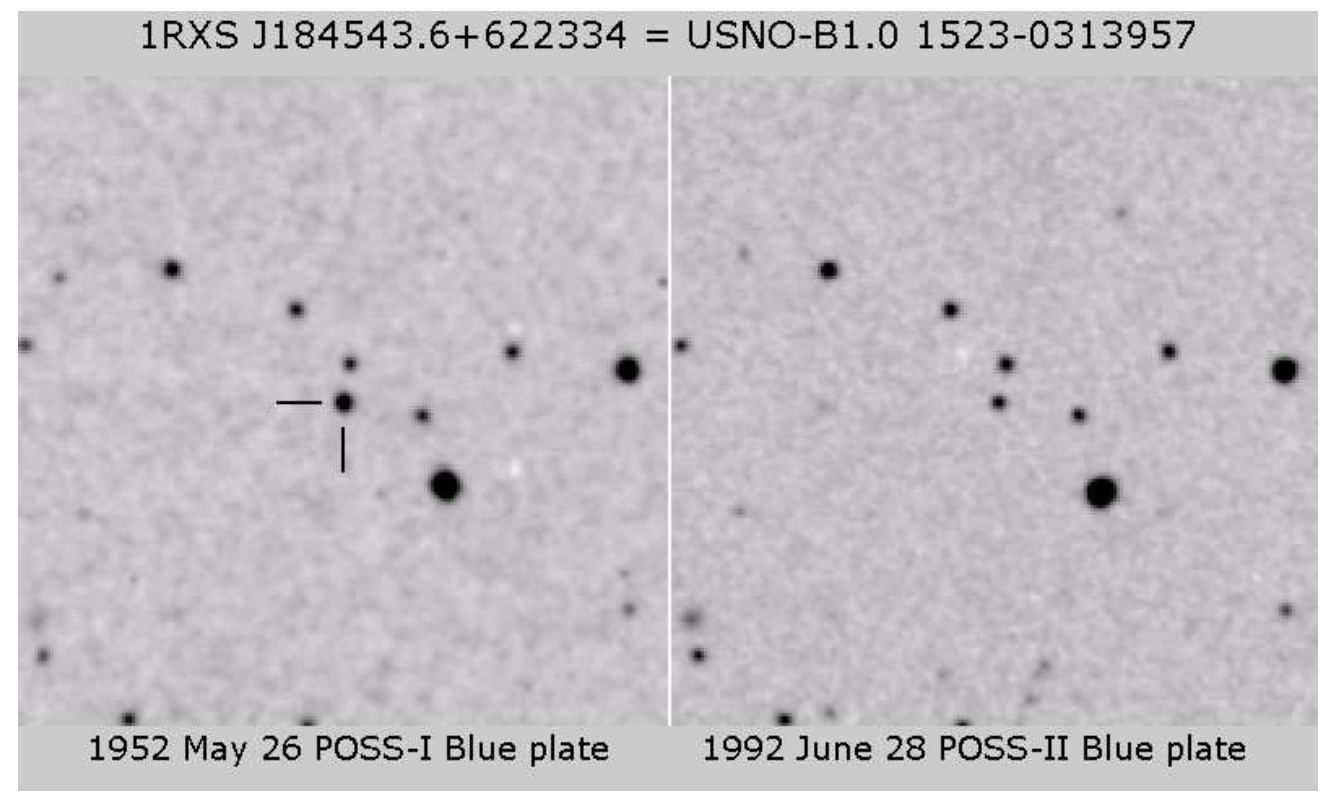

Figure 6. Finder chart of the source 1RXS J184543.6+622334 $\left(200^{\prime \prime} \times 200^{\prime \prime}\right)$.

1RXS J185310.0+594509 = USNO-B1.0 1497-0258111: R.A.= 18:53:09.556, Dec. $=+59: 45: 07.22$, J2000, proper motion: (16, 12) mas/year. Outburst on the infrared plate: $\mathrm{B} 1=17.75, \mathrm{R} 1=18.66, \mathrm{~B} 2=17.87, \mathrm{R} 2=18.51, \mathrm{I}=15.44$. X-ray hardness $(\mathrm{HR} 1=0.40 \pm 0.21, \mathrm{HR} 2=0.29 \pm 0.25)$ also does not contradict the hypothesis of the magnetic nature of this CV. The finding chart of the object is presented on Fig. 7 .

1RXS J192926.6+202038 = USNO-B1.0 1103-0421031: R.A.= 19:29:27.820, Dec. $=+20: 20: 35.36$, J2000, proper motion: $(10,10)$ mas/year. Resides in a very dense star field (galactic latitude $\left.b=+1.2^{\circ}\right)$. X-ray hardness $(\mathrm{HR} 1=1.00 \pm 0.34, \mathrm{HR} 2=0.22 \pm 0.39$ ) is close to similar parameters for the dwarf nova 1RXS J231935.0+364705 (1.00 \pm 0.09 and $0.47 \pm 0.16$, respectively). The finding chart of the object is presented on Fig. 8.

1RXS J194151.4+752621 = USNO-B1.0 1654-0092633: R.A.= 19:41:51.597, Dec. $=+75: 26: 12.81$, J2000, proper motion: $(-6,12)$ mas/year. X-ray hardness $(\mathrm{HR} 1=$ $0.88 \pm 0.16, \mathrm{HR} 2=0.36 \pm 0.19)$ which is typical for dwarf novae along with rather large amplitude of variability allow us to classify this object confidently as a cataclysmic variable of U Gem type. The finding chart of the object is presented on Fig. 9. 


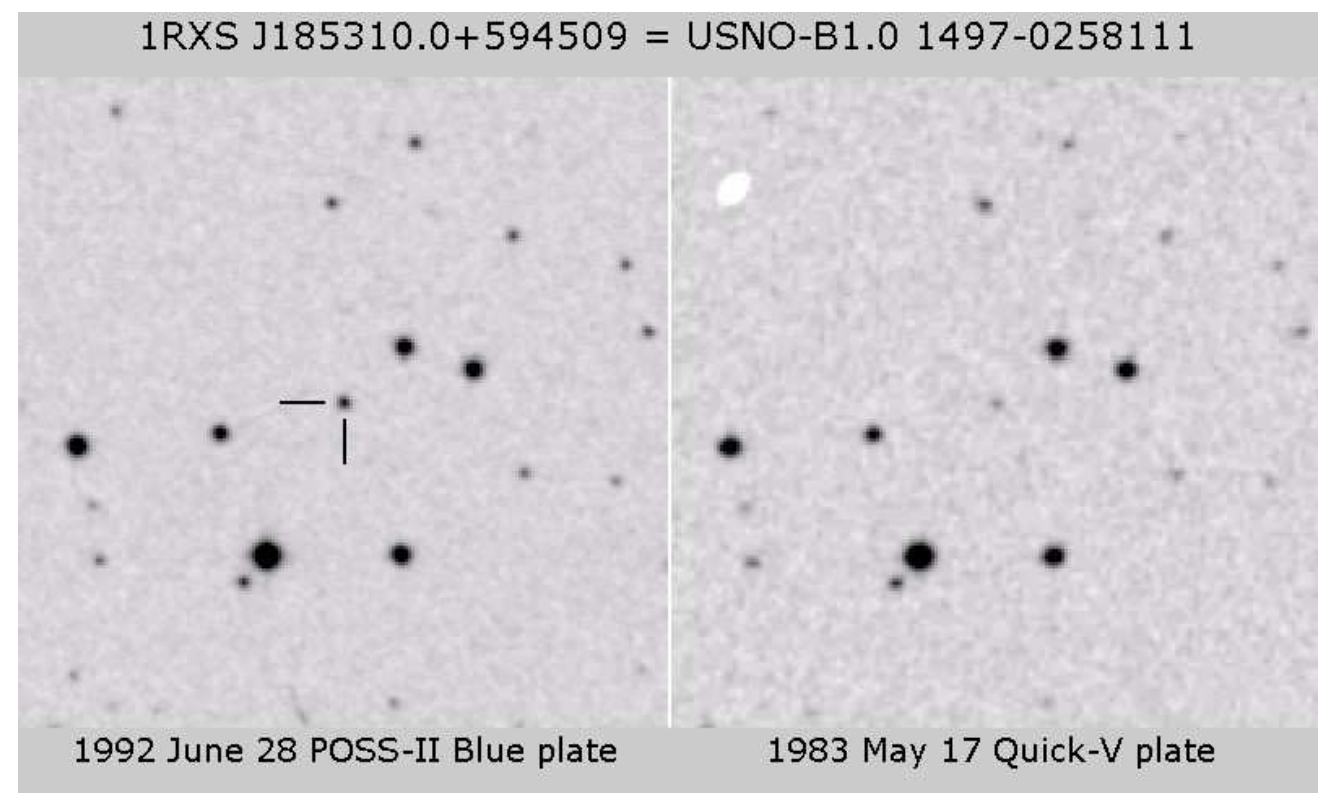

Figure 7. Finder chart of the source 1RXS J185310.0+594509 $\left(200^{\prime \prime} \times 200^{\prime \prime}\right)$. Left - Blue POSS-II plate of 1992, right - photovisual 1983 plate.

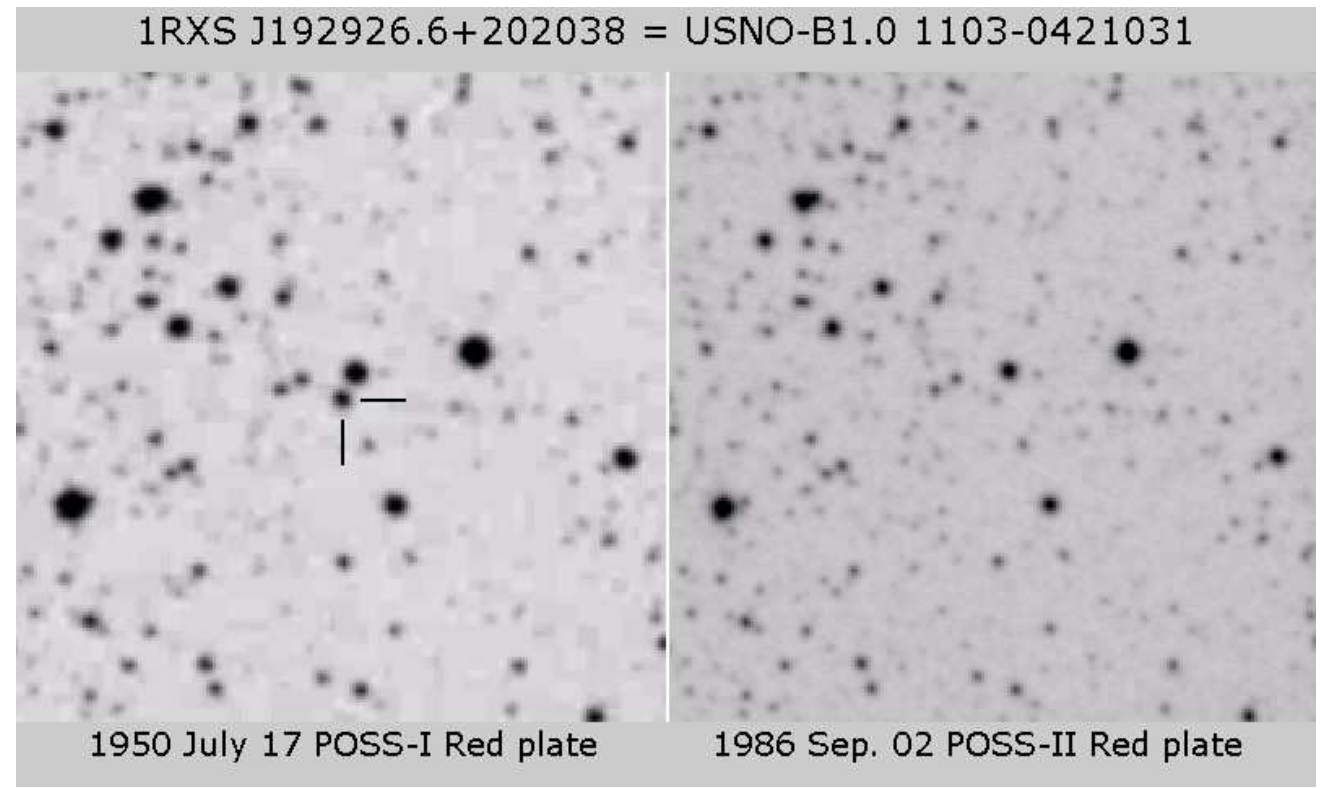

Figure 8. Finder chart of the source 1RXS J192926.6+202038 $\left(200^{\prime \prime} \times 200^{\prime \prime}\right)$.

1RXS J222335.6+074515 = USNO-B1.0 0977-0743560: R.A.= 22:23:34.159, Dec. $=+07: 45: 19.27, \mathrm{~J} 2000$, proper motion: $(-12,-18)$ mas/year. We have downloaded 52 images of this variable star by the NEAT project from the SkyMorph site (Pravdo et al., 2005) covering 4 years of observations (June 2001 - September 2005). The corresponding light curve is shown at Fig. 11. The star is exhibiting frequent outbursts fading to minimum 


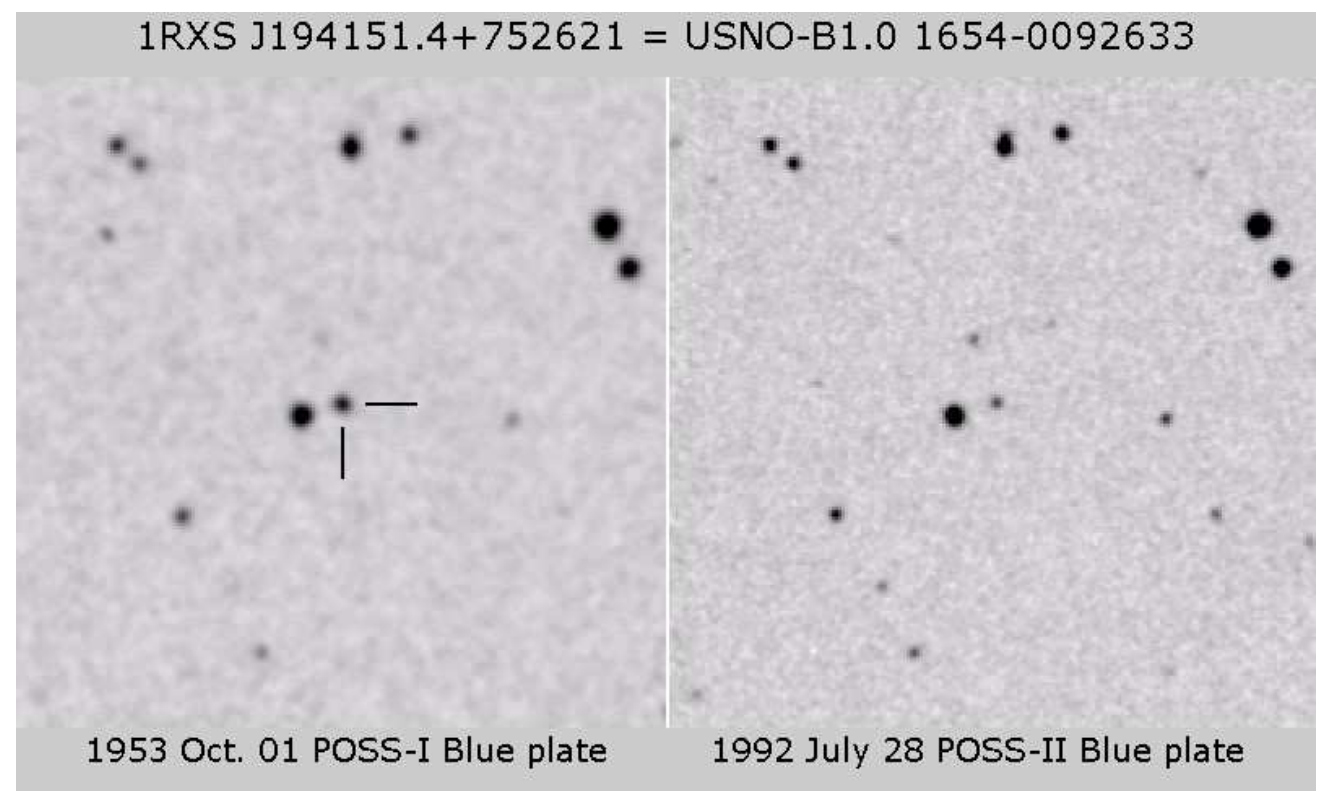

Figure 9. Finder chart of the source 1RXS J194151.4+752621 $\left(200^{\prime \prime} \times 200^{\prime \prime}\right)$.

and rising by more than $2.5 \mathrm{~m}$ within less than 10 days. This object is likely a dwarf nova of UGSU or UGZ subtype. The finding chart of the object is presented on Fig. 10.

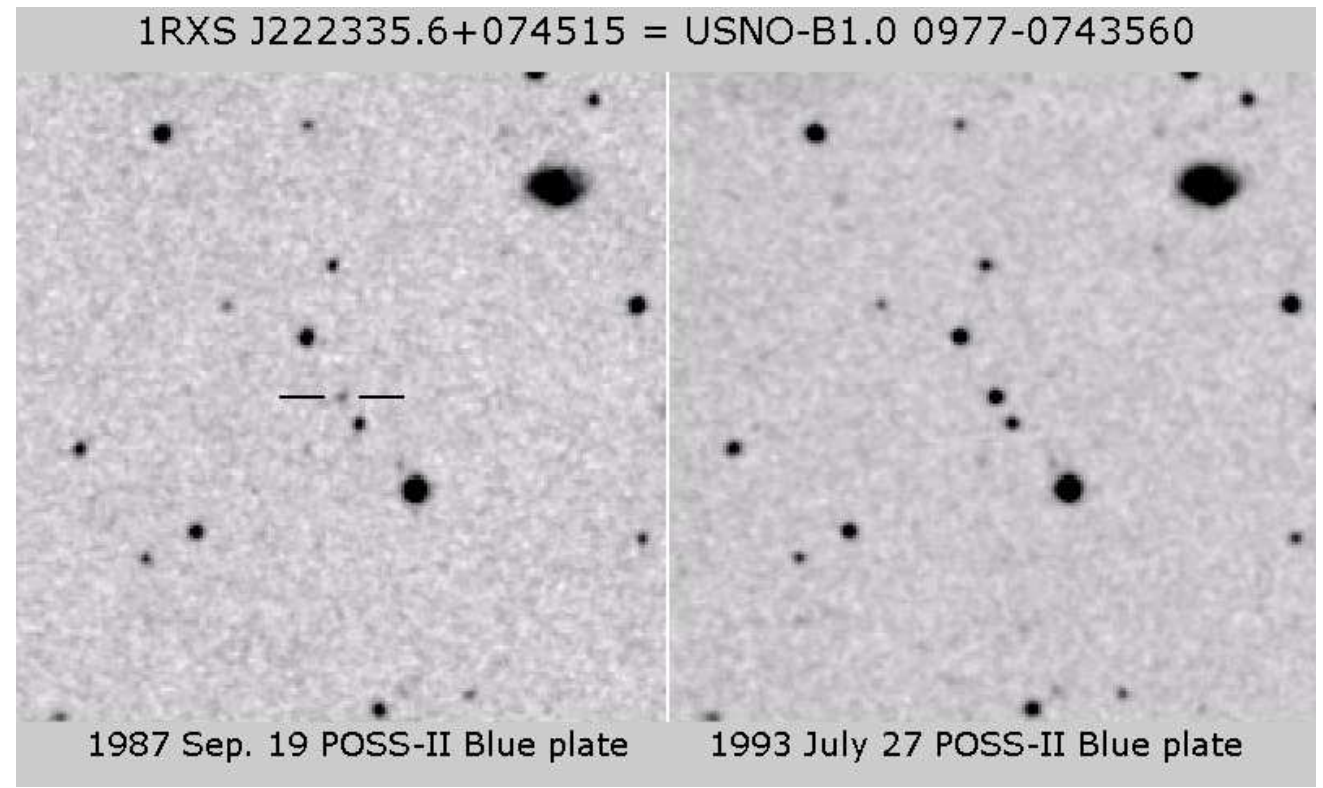

Figure 10. Finder chart of the source 1RXS J222335.6+074515 $\left(200^{\prime \prime} \times 200^{\prime \prime}\right)$. 


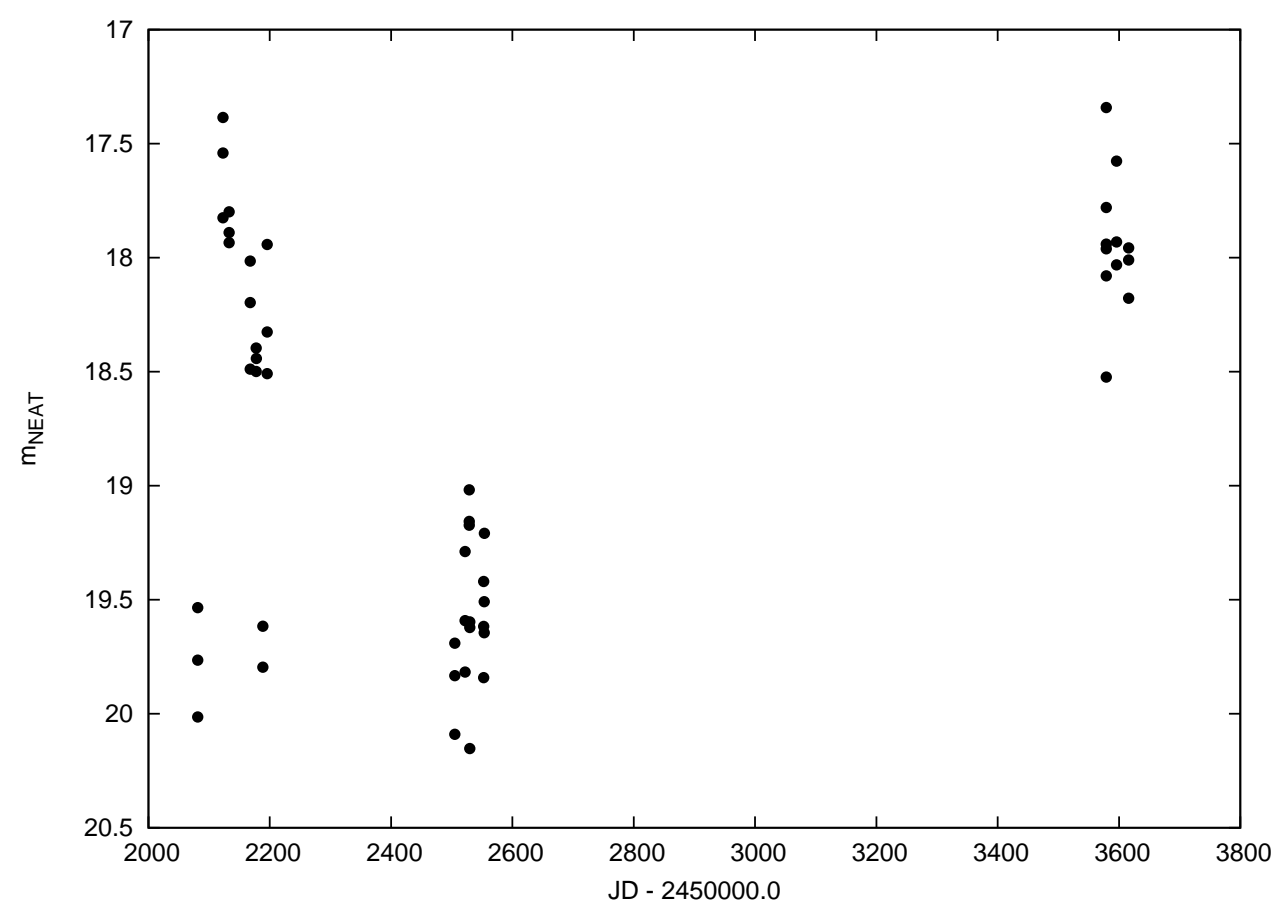

Figure 11. Light curve of the source 1RXS J222335.6+074515 based on the observations by NEAT.

\section{RED/WHITE DWARF COMMON PROPER MOTION PAIR}

The search for the cataclysmic variables in the USNO-B1.0 catalog has resulted in an unexpected by-product - the discovery of the pair "white dwarf - red dwarf" with the common proper motion just below $0.2^{\prime \prime}$ per year which could have otherwise remained unnoticed for long. This binary system is an optical counterpart of the 1RXS J052436.9-053525 X-ray source. Only one member of the pair with a proper motion is listed in the UCAC2 (Zacharias et al., 2004) and UCAC3 (Zacharias et al., 2010) catalogs. Both stars are present in 2MASS catalog but without a proper motion. The USNO-B1.0 catalog formally includes both stars, but in fact their data are mixed with each other and the proper motions are given erroneously. The position of the red dwarf on the 1st epoch plates (POSS-I) taken in December of 1955 almost coincides with the position of white dwarf on the 2nd epoch images (red plate of 1994 and infrared one of 2002). As a result the USNO-B1.0 catalog contains the record number 0844-0057342 with the following coordinates and peculiar color indices: R.A.=05:24:36.54, Dec. $=-05: 35: 00.9, \mathrm{~B} 1=12.77, \mathrm{R} 1=15.50, \mathrm{~B} 2=12.23, \mathrm{R} 2=14.96, \mathrm{I}=12.76$.

Visual comparison of 1st and 2nd epoch DSS plates has shown that two stars with separation about $7^{\prime \prime}$ at $\mathrm{PA}=159^{\circ}$ are moving together with virtually identical proper motions 
about (-20, -160) mas/year along the Right Ascension and Declination respectively, that is the pair's common proper motion is directed almost along the vector joining the two stars. The data from the 2MASS catalog for both stars agree with the hypothesis that this pair is a gravitationally bound system of the white and red dwarfs at the same distance of about 40 pc:

2MASS 05243648-0535175: $J=12.103 \pm 0.027, H=11.535 \pm 0.023, K_{s}=11.116 \pm 0.024$

2MASS 05243627-0535103: $J=14.213 \pm 0.051, H=14.260 \pm 0.058, K_{s}=14.446 \pm 0.080$

Reid et al. (2007) are providing M5.0 spectral class and distance of 37.9 pc for the red dwarf but do not mention the second component. One can estimate the distance between two components in the sky plane (lower limit on the size of the binary orbit) as 292 A.U. With the mass of the system equal to that of the Sun orbital period would be no less than 5000 years. That agrees well with the absence of the detectable orbital motion of components during 39 years having passed between 1st and 2nd epoch DSS plates.

\section{SUMMARY}

Using rather simple search method over the publicly available data we have discovered eight new cataclysmic variables and two Optically Violent Variable quasar candidates as well as a "white dwarf - red dwarf" common propper motion pair among the previously unidentified sources of $1 \mathrm{RXS}$ catalog. This result demonstrates the large potential of the Virtual Observatory concept in identifying peculiar objects. Variable objects reported in this paper are in need of follow-up observations to confirm and refine their classification. They are all accessible for observatories in the Northern hemisphere. The color combined finder charts of the new variables may be found at http://hea.iki.rssi.ru/ denis/CV-USNO.html.

\section{ACKNOWLEDGMENTS}

Authors are thankful to Dr. N. N. Samus for providing the opportunity to work with the archival photographic plates of the Moscow collection at SAI MSU. We are grateful to E. Angelakis and the anonymous referee for reviewing the English and Russian versions of this manuscript. D.D. would like to thank the Program of support of the leading scientific schools (grant NSh-5069.2010.2). K.S. is a member of the International Max Planck Research 
School (IMPRS) for Astronomy and Astrophysics at the universities of Bonn and Cologne. The work has made an intensive use of Palomar Observatory Sky Survey plates obtained by the California Institute of Technology with the funds from NSF, NGS, Sloan Foundation, Samuel Oschin Foundation and Eastman Kodak Corporation. While preparing the article we have made use of the SIMBAD database operated at CDS, Strasbourg, France, and the Variable Star Index (VSX) maintained by the American Association of Variable Star Observers.

1. Y. Bai, Y. Chen, X.-T. He, et al., Chin. J. of Astron. and Astrophys., 7, 375 (2007).

2. J. J. Condon, W. D. Cotton, E. W Greisen, et al., Astron. J. 115, 1693 (1998).

3. D. V. Denisenko, M. N. Pavlinsky, R. A. Sunyaev, et al., Astron. Letters, 32, 252 (2006).

4. D. V. Denisenko, Peremennye Zvezdy (Prilozhenie), 8, 25 (2008).

5. D. V. Denisenko T. V. Kryachko, B. L. Satovskiy, Astron. Telegram 1640 (2008).

6. D. V. Denisenko T. V. Kryachko, B. L. Satovskiy, Astron. Telegram 2014 (2009).

7. D. V. Denisenko, Astron. Telegram 2282 (2009).

8. A. J. Drake, S. G. Djorgovski, A. Mahabal, et al., Astrophys. J. 696, 870 (2009).

9. B. T. Gänsicke, T.R. Marsh, A. Edge, et al., MNRAS, 361, 141 (2005).

10. X.-T. He, J.-H. Wu, Q.-R. Yuan, et al., Astron. J., 121, 1863 (2001).

11. D. G. Monet, S. E. Levine, B. Canzian, et al., Astron. J., 125, 984 (2003).

12. C. Motch, F. Haberl, P. Guillout, et al., Astron. Astrophys. 307, 459 (1996).

13. S. H. Pravdo, D. R. Rabinovitz, E. F. Helin, et al., http://skys.gsfc.nasa.gov/skymorph/ skymorph.html (2005).

14. I. N. Reid, K. L. Cruz, P. R. Allen, Astron. J., 133, 2825 (2007).

15. N. N. Samus, O. V. Durlevich, et al., 2009yCat....102025S (2007-2009).

16. M. F. Skrutskie, R. M. Cutri, R. Stiening, et al., Astron. J., 131, 1163 (2006).

17. B. J. Teegarden, S. H. Pravdo, M. Hicks, et al., Astrophys. J. 589, L51 (2003).

18. F. Verbunt, W. H. Bunk, H. Ritter, and E. Pfeffermann, Astron. Astrophys. 327, 602 (1997).

19. W. Voges, B. Aschenbach, T. Boller, et al., Astron. Astrophys. 349, 389 (1999).

20. W. Voges, B. Aschenbach, T. Boller, et al., IAU Circ. 7432 (2000).

21. C. Watson, A. A Henden, A. Price // The Society for Astronomical Sciences 25th Annual 
Symposium on Telescope Science. Held May 23-25, 2006, at Big Bear, CA. Published by the Society for Astronomical Sciences., p.47 (2006).

22. P. Wils, B. T. Gänsicke, A. J. Drake, J. Southworth, MNRAS, 402, 436 (2009).

23. N. Zacharias, S. E. Urban, M. I. Zacharias, et al., Astron. J., 127, 3043 (2004).

24. N. Zacharias, C. Finch, T. Girard, et al., Astron. J., 139, 2184 (2010).

25. F.-J. Zickgraf, D. Engels, H.-J. Hagen, et al., Astron. Astrophys. 406, 535 (2003). 